\title{
Improved glycaemia in high-fat-fed neprilysin-deficient mice is associated with reduced DPP-4 activity and increased active GLP-1 levels
}

\author{
Joshua R. Willard ${ }^{1}$ • Breanne M. Barrow ${ }^{1}$ • Sakeneh Zraika ${ }^{1,2}$
}

Received: 25 October 2016 / Accepted: 16 November 2016/Published online: 8 December 2016

(C) Springer-Verlag Berlin Heidelberg 2016

\begin{abstract}
Aim/hypothesis Neprilysin, a widely expressed peptidase, is upregulated in metabolically altered states such as obesity and type 2 diabetes. Like dipeptidyl peptidase-4 (DPP-4), neprilysin can degrade and inactivate the insulinotropic peptide glucagon-like peptide-1 (GLP-1). Thus, we investigated whether neprilysin deficiency enhances active GLP-1 levels and improves glycaemia in a mouse model of high fat feeding. Methods $\mathrm{Nep}^{+/+}$and $\mathrm{Nep}^{-/-}$mice were fed a $60 \%$ fat diet for 16 weeks, after which active GLP-1 and DPP-4 activity levels were measured, as were glucose, insulin and C-peptide levels during an OGTT. Insulin sensitivity was assessed using an insulin tolerance test.

Results High-fat-fed $\mathrm{Nep}^{-/-}$mice exhibited elevated active GLP-1 levels (5.8 \pm 1.1 vs $3.5 \pm 0.8 \mathrm{pmol} / 1, p<0.05)$ in association with improved glucose tolerance, insulin sensitivity and beta cell function compared with high-fat-fed $\mathrm{Nep}^{+/+}$mice. In addition, plasma DPP-4 activity was lower in high-fat-fed $\mathrm{Nep}^{-/-}$mice $\left(7.4 \pm 1.0\right.$ vs $10.7 \pm 1.3 \mathrm{nmol} \mathrm{ml}^{-1} \mathrm{~min}^{-1}$, $p<0.05)$. No difference in insulin:C-peptide ratio was observed between $\mathrm{Nep}^{-/-}$and $\mathrm{Nep}^{+/+}$mice, suggesting that improved glycaemia does not result from changes in insulin clearance.
\end{abstract}

Joshua R. Willard and Breanne M. Barrow contributed equally to this study.

Sakeneh Zraika

zraikas@uw.edu

1 Veterans Affairs Puget Sound Health Care System, 1660 South Columbian Way (151), Seattle, WA 98108, USA

2 Division of Metabolism, Endocrinology \& Nutrition, Department of Medicine, University of Washington, Seattle, WA, USA
Conclusions/interpretation Under conditions of increased dietary fat, an improved glycaemic status in neprilysindeficient mice is associated with elevated active GLP-1 levels, reduced plasma DPP-4 activity and improved beta cell function. Thus, neprilysin inhibition may be a novel treatment strategy for type 2 diabetes.

Keywords DPP-4 · GLP-1 - Glucose tolerance $\cdot$ High fat diet $\cdot$ Insulin secretion $\cdot$ Mme $\cdot$ Mouse $\cdot \mathrm{Nep} \cdot$ Neprilysin
Abbreviations
DPP-4 Dipeptidyl peptidase-4
GLP-1 Glucagon-like peptide-1
ITT Insulin tolerance test

\section{Introduction}

Neprilysin (encoded by Nep, also known as Mme) is a widely expressed plasma membrane peptidase with increased levels in metabolically altered states [1-3]. We and others have demonstrated that reducing neprilysin activity may be beneficial via improving insulin secretion [4] and sensitivity [5-7].

Glucagon-like peptide-1 (GLP-1) is a substrate for both dipeptidyl peptidase-4 (DPP-4) and neprilysin. DPP-4 removes the first two $\mathrm{N}$-terminal amino acids, rendering GLP-1 non-insulinotropic, although it can still bind to its receptor $[8,9]$. Neprilysin cleaves active GLP-1 at six sites in the central and C-terminal regions $[10,11]$, thereby eliminating its ability to bind to the GLP-1 receptor. Up to $50 \%$ of exogenously infused GLP-1 may be cleaved by neprilysin [12], and the combination of neprilysin and DPP-4 inhibition is more effective at maintaining active GLP-1 levels and enhancing insulin secretion compared with inhibition of 
DPP-4 alone [12]. However, it is unknown whether neprilysin inhibition affects DPP-4 activity. In this study, we sought to determine whether neprilysin inhibition preserves endogenous active GLP-1 levels in a model of high fat feeding and, if so, whether it is associated with reduced DPP-4 activity and improved glucose homeostasis.

\section{Methods}

\section{Animals and diets}

Ten-week-old C57BL/6.Nep $\mathrm{N}^{+/+}$and C57BL/6.Nep ${ }^{-/-}$ male mice (originally from B. Lu, Children's Hospital, Harvard Medical School, Boston, MA, USA [13]) were fed diets containing either $10 \%$ or $60 \%$ fat (Research Diets, New Brunswick, NJ, USA) for 16 weeks. Mice were housed two per cage, with a $12 \mathrm{~h}$ light/12 h dark cycle, and food and water ad libitum. Body weight, food intake and fed plasma glucose levels were assessed weekly. The study was approved by the Institutional Animal Care and Use Committee of VA Puget Sound Health Care System.

\section{Insulin and glucose tolerance tests}

Following the 16 week feeding period, i.p. insulin tolerance tests $(1.0 \mathrm{U} / \mathrm{kg}$; ITTs) were performed in conscious mice after a $4 \mathrm{~h}$ fast. Tail vein blood was collected at 0, 15, 30, 45 and $60 \mathrm{~min}$ post insulin administration for glucose measurement. After 2 days, OGTTs ( $2 \mathrm{~g} / \mathrm{kg}$ ) were performed in anaesthetised mice fasted for $16 \mathrm{~h}$. Plasma was collected in heparinised tubes at 0,10, 20, 30, 60, 90 and 120 min post glucose bolus for measuring glucose, insulin and C-peptide levels and at 10 min post glucose bolus for measuring active GLP-1 levels. To prevent degradation of GLP-1 in plasma, $500 \mathrm{kU} / \mathrm{ml}$ aprotinin, $3.2 \mathrm{mmol} / 1 \mathrm{EDTA}$ and $0.01 \mathrm{mmol} / 1$ diprotin A were added to the blood collected for GLP-1 measurement. Plasma samples were stored at $-30^{\circ} \mathrm{C}$ prior to assay.

\section{Glucose, insulin, C-peptide and GLP-1 assays}

For weekly bleeds and OGTTs, plasma glucose levels were determined using the glucose oxidase method. For ITTs, blood glucose levels were measured using an AlphaTRAK2 glucometer (Abbott Laboratories, Abbott Park, IL, USA). Plasma insulin, C-peptide and active GLP-1 levels were measured using the Mouse Ultrasensitive Insulin, Mouse C-Peptide and GLP-1 (Active 7-36) ELISAs (Alpco, Salem, $\mathrm{NH}, \mathrm{USA})$, respectively.

\section{Neprilysin and DPP-4 activity assays}

Neprilysin activity was determined in plasma samples taken from mice following 2, 6, 10, 12, 14 and 16 weeks of feeding. Lateral saphenous vein blood from conscious, fed mice was collected in heparinised tubes and centrifuged to obtain plasma, which was stored at $-30^{\circ} \mathrm{C}$ prior to assay. In the neprilysin activity assay, glutaryl-Ala-Ala-Phe-4methoxy-2-naphthylamine is broken down by neprilysin in plasma to phe-4-methoxy-2-naphthylamine, and then by aminopeptidase $\mathrm{M}$ to the fluorescent product methoxy-2naphthylamine [14]. Each plasma sample was assayed in both the absence and presence of a specific neprilysin inhibitor (DL-thiorphan, Sigma-Aldrich, St Louis, MO, USA) to distinguish neprilysin activity from non-specific endopeptidase activity. Fluorescence was compared against a methoxy-2naphthylamine standard curve.

DPP-4 activity was determined in plasma samples obtained from mice immediately after the OGTT. Arterial (carotid) blood was collected in heparinised tubes and centrifuged to obtain plasma, which was stored at $-30^{\circ} \mathrm{C}$ prior to assay. In this assay, DPP4 cleaves non-fluorescent H-Gly-Pro-7-amino-4methylcoumarin to generate fluorescent 7-amino-4methylcoumarin [15]. Plasma DPP-4 activity was compared against a 7-amino-4-methylcoumarin standard curve.

\section{Effect of ex vivo and in vitro inhibition of neprilysin on DPP-4 activity}

Ex vivo assay Arterial (carotid) blood from low-fat and high-fat-fed $\mathrm{Nep}^{+/+}$mice was collected in heparinised tubes immediately after the OGTT. Blood was centrifuged to obtain plasma, which was stored at $-30^{\circ} \mathrm{C}$ prior to the following treatment. Plasma from each mouse was divided into two samples: one was incubated with the neprilysin inhibitor DL-thiorphan $(20 \mu \mathrm{mol} / \mathrm{l})$ and the other was incubated with vehicle $(50 \mathrm{mmol} / 1$ Tris, $\mathrm{pH} 7.5)$ for $1 \mathrm{~h}$ at $37^{\circ} \mathrm{C}$. Neprilysin and DPP-4 activities were then determined as described above. DL-Thiorphan was used at $20 \mu \mathrm{mol} / 1$ because this concentration was found to maximally inhibit plasma neprilysin activity in a dose-response analysis (data not shown). DL-thiorphan did not affect DPP-4 activity directly, as determined by measuring DPP-4 activity in plasma from low-fat and high-fat-fed $\mathrm{Nep}^{-/-}$mice after incubation with $20 \mu \mathrm{mol} / \mathrm{l}$ DL-thiorphan (or vehicle). In addition, DPP-4 levels in plasma from low-fat-fed mice were above the limit of detection for this assay, as determined by measuring DPP-4 activity in plasma incubated with varying concentrations of the DPP-4 inhibitor diprotin A $(0.01,0.1$, $0.2,0.5$ and $1 \mathrm{mmol} / \mathrm{l}$ ) or vehicle. All diprotin A concentrations reduced DPP-4 activity; $1 \mathrm{mmol} / 1$ diprotin A reduced DPP-4 activity by $93 \%$ compared with vehicle. 
In vitro assay In separate studies, recombinant human neprilysin $(0.01 \mu \mathrm{mol} / 1$; ACROBiosystems, Newark, DE, USA) was incubated with $50 \mu \mathrm{mol} / 1 \mathrm{DL}$-thiorphan or vehicle (50 mmol/1 Tris, $25 \mu \mathrm{mol} / 1 \mathrm{ZnCl}_{2}, \mathrm{pH} 7.5$ ) for $1 \mathrm{~h}$ at $37^{\circ} \mathrm{C}$. Recombinant human DPP-4 (R\&D Systems, Minneapolis, MN, USA) was then added at a final concentration of 0.01 , 0.05 or $0.1 \mu \mathrm{mol} / 1$, and incubated for $1 \mathrm{~h}$ at $37^{\circ} \mathrm{C}$. Molar ratios of 1:1, 1:5 and 1:10 neprilysin to DPP-4 were tested, with the 1:5 and 1:10 ratios approximating those documented in plasma from lean and obese humans [3, 16, 17]. Samples were assayed for neprilysin and DPP-4 activities as described above. Specificity controls were included in which samples containing only DPP-4 were assayed for neprilysin activity and samples containing only neprilysin were assayed for DPP-4 activity. As there was no difference in DPP-4 activity following incubation of $0.1 \mu \mathrm{mol} / 1 \mathrm{DPP}-4$ with $50 \mu \mathrm{mol} / 1$ DL-thiorphan or vehicle for $1 \mathrm{~h}$ at $37^{\circ} \mathrm{C}$, non-specific inhibition of DPP-4 activity by DL-thiorphan could be ruled out. As a positive control, inhibition of DPP-4 activity using our experimental paradigm was demonstrated by incubating DPP-4 $(0.01,0.05$ or $0.1 \mu \mathrm{mol} / \mathrm{l})$ and $0.01 \mu \mathrm{mol} / 1$ neprilysin with $2 \mathrm{mmol} / \mathrm{l}$ diprotin A.

\section{Statistical analysis}

Data are presented as means \pm SEM. Beta cell function was calculated as the incremental AUC for insulin over 0-30 min divided by the incremental AUC for glucose over 0-30 min divided by the fasting insulin level. Statistical significance was determined using ANOVA with post hoc analysis or the Mann-Whitney $U$ test if data were not normally distributed. A $p$ value of $<0.05$ was considered statistically significant.

\section{Results}

\section{Weight gain, food intake and fed glucose levels}

Mice fed a high-fat diet gained significantly more weight regardless of genotype (Fig. 1a). However, $\mathrm{NeP}^{-/-}$mice gained less weight than $\mathrm{Nep}^{+/+}$mice, beginning after 3 weeks on the high-fat diet and from 13 weeks onward on the low-fat diet. Food intake was similar between $\mathrm{Nep}^{-/-}$and $\mathrm{Nep}^{+/+}$mice on both the high- and low-fat diets (data not shown).

Over 16 weeks, fed glucose levels were comparable and stable between $\mathrm{Nep}^{-/-}$and $\mathrm{Nep}^{+/+}$mice on a low-fat diet (Fig. 1b). In contrast, $\mathrm{Nep}^{+/+}$mice fed a high-fat diet displayed progressively elevated glucose levels over time compared with mice on a low-fat diet. $\mathrm{Nep}^{-1-}$ mice on a high-fat diet also had elevated glucose levels, although a progressive increase was not observed and levels remained significantly lower than in $\mathrm{Nep}^{+/+}$mice on a high-fat diet.

\section{OGTTs and ITTs}

During the OGTT, glucose and insulin levels were comparable between genotypes in mice on a low-fat diet, but were higher in $\mathrm{Nep}^{+/+}$than in $\mathrm{Nep}^{-/-}$mice on a high-fat diet (Fig. 1c, d). The insulin:C-peptide ratio was calculated to determine whether high-fat-fed $\mathrm{Nep}^{-/-}$mice displayed reduced insulin clearance; however, no significant increase in the ratio was seen in $\mathrm{Nep}^{-/-}$vs $\mathrm{Nep}^{+/+}$mice (data not shown).

Glucose clearance during the ITT was comparable in mice on a low-fat diet regardless of genotype (Fig. 1e). Mice on a high-fat diet displayed poorer insulin sensitivity than those on a low-fat diet; however, $\mathrm{Nep}^{-/-}$mice were more insulin sensitive than $\mathrm{Nep}^{+/+}$mice.

To determine beta cell function, we investigated the relationship between OGTT insulin responses and fasting insulin levels (a surrogate for insulin sensitivity). Beta cell function was reduced in high-fat-fed vs low-fat-fed $\mathrm{Nep}^{+/+}$mice, but was significantly greater in high-fat-fed $\mathrm{Nep}^{-/-} \mathrm{vs} \mathrm{Nep}^{+/+}$mice (Fig. 1f).

\section{Neprilysin and DPP-4 activities and active GLP-1 levels in vivo}

Plasma neprilysin activity was undetectable in $\mathrm{Nep}^{-/}$mice (data not shown), while it increased in $\mathrm{Nep}^{+/+}$mice after 10 weeks of high-fat feeding (Fig. 2a). After 16 weeks of feeding, plasma DPP-4 activity was comparable in mice fed a low-fat diet regardless of genotype (Fig. 2b). With a high-fat diet, both $\mathrm{Nep}^{+++}$and $\mathrm{Nep}^{-1-}$ mice displayed elevated DPP-4 activity; however, levels were significantly lower in $\mathrm{Nep}^{-/-}$ than $\mathrm{Nep}^{+/+}$mice.

Active GLP-1 levels were comparable in mice fed a low-fat diet regardless of genotype (Fig. $2 \mathrm{c}$ ). In contrast, $\mathrm{Nep}^{-/-}$mice on a high-fat diet had higher active GLP-1 levels than both $\mathrm{Nep}^{+/+}$mice on a high-fat diet and $\mathrm{Nep}^{-/-}$mice on a low-fat diet.

\section{Effect of ex vivo and in vitro inhibition of neprilysin on DPP-4 activity}

To determine whether pharmacological inhibition of neprilysin activity could reduce DPP-4 activity in plasma (as seen with genetic ablation of Nep in high-fat-fed mice), the neprilysin inhibitor DL-thiorphan (or vehicle) was incubated ex vivo with plasma from $\mathrm{Nep}^{+/+}$mice fed a low- or high-fat diet for 16 weeks and then DPP-4 activity measured. DL-Thiorphan inhibited neprilysin activity by $95 \pm 2 \%$ and $91 \pm 6 \%$ in plasma from low-fat and high-fat-fed $\mathrm{Nep}^{+/+}$mice, respectively (Fig. 3a). DPP-4 activity was greater in vehicle-treated plasma from high-fat-fed vs low-fat-fed mice (Fig. 3b). While DL-thiorphan treatment did not alter DPP-4 activity in plasma from low-fat-fed mice, it significantly reduced DPP-4 activity in plasma from 
Fig. 1 Neprilysin deficiency improves the glycaemic status of high-fat-fed mice. (a) Body weight and (b) fed glucose levels over time in $\mathrm{Nep}^{+/+}$and $\mathrm{Nep}^{-/-}$ mice fed a low- or high-fat diet for 16 weeks. Plasma (c) glucose and (d) insulin levels during an OGTT, (e) blood glucose levels during an ITT and (f) beta cell function after 16 weeks of low- or high-fat feeding. Sequential blood samples were taken from the same mouse for each measure. Data are means \pm SEM. (a, b) $n=19-22$ per group. (c-f) $n=6-18$ per group. (a-e) White squares, $\mathrm{Nep}^{+/+} /$low-fat diet; black squares, $\mathrm{Nep}^{+/+} /$high-fat diet; white circles, $\mathrm{Nep}^{-/-} /$low-fat diet; black circles, $\mathrm{Nep}^{-/} /$high-fat diet. (f) White bars, low-fat diet; black bars, high-fat diet. * $p<0.05$ vs $\mathrm{Nep}^{-/-}$. iAUC, incremental AUC; ins, insulin; glu, glucose a
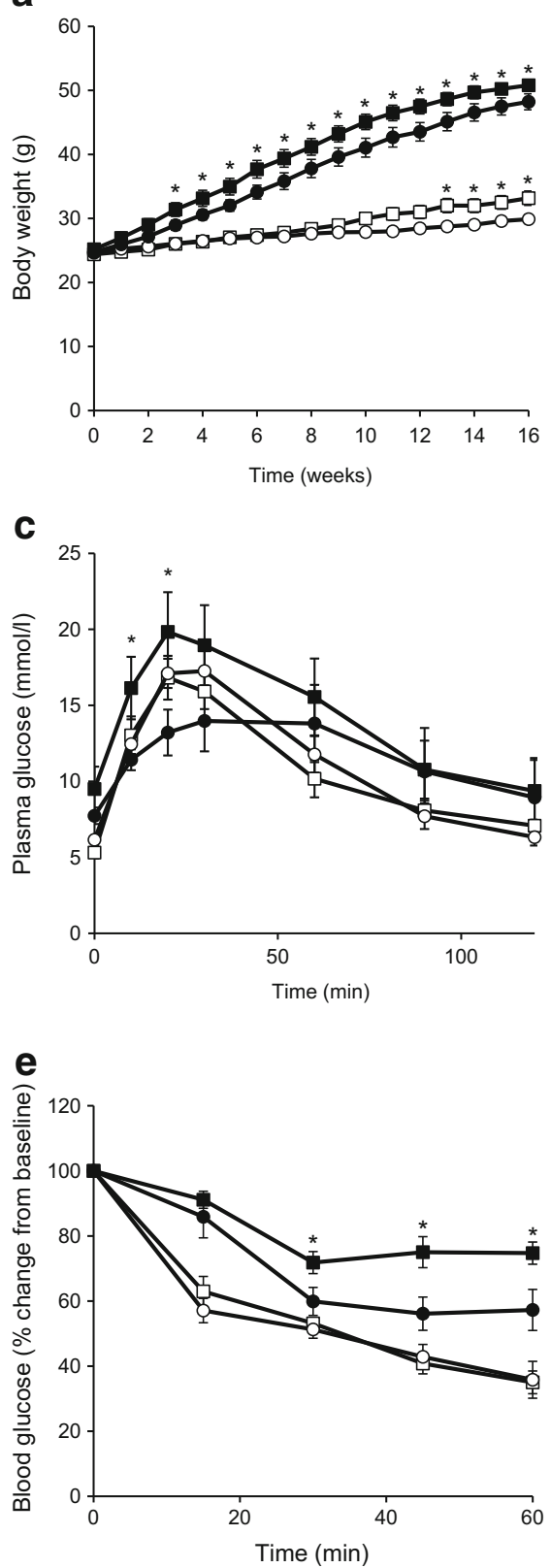

b

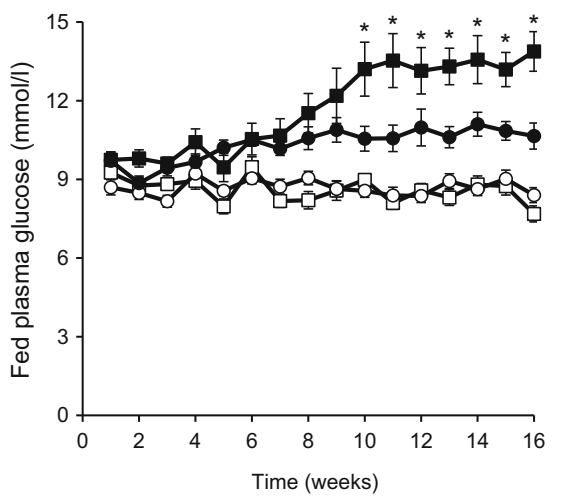

d

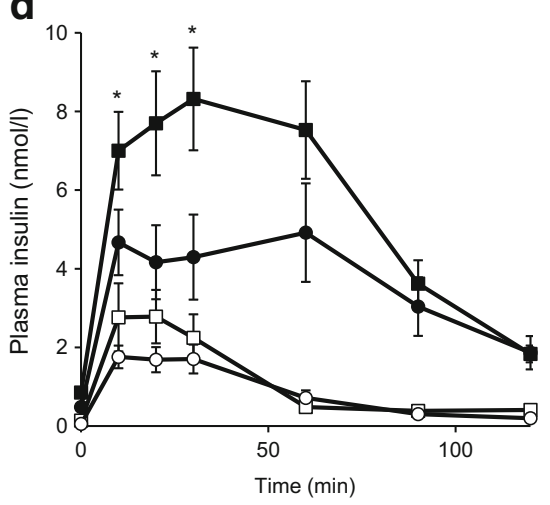

f

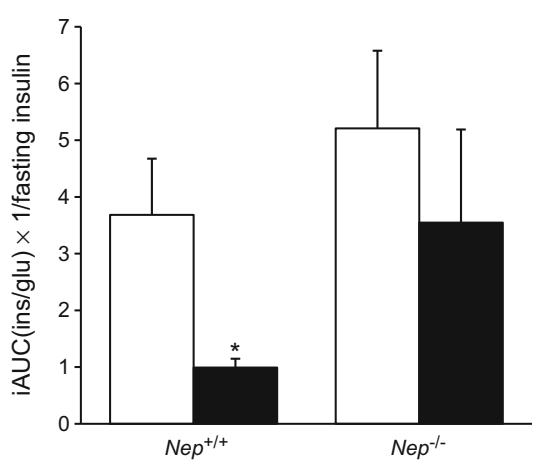

high-fat-fed mice (Fig. 3b). The latter finding is consistent with the observation of lower in vivo DPP-4 activity in plasma from high-fat-fed $\mathrm{Nep}^{-/-}$vs $\mathrm{Nep}^{+/+}$mice.

To determine whether neprilysin directly inhibits DPP-4 activity, recombinant neprilysin was treated with the inhibitor DL-thiorphan and then incubated with recombinant DPP-4 in vitro at a neprilysin:DPP-4 molar ratio of $1: 5$ or $1: 10$, to match proportions found in plasma from lean and obese humans [3, 16, 17]. Measurement of DPP-4 activity showed that DL-thiorphan treatment resulted in $75 \pm 2 \% \quad(n=3$, $p<0.05)$ and $84 \pm 4 \%(n=3, p<0.05)$ inhibition of neprilysin activity in samples containing neprilysin:DPP-4 molar ratios of 1:5 and 1:10, respectively, but had no effect on DPP-4 activity (Fig. 3c). Even at a molar ratio of 1:1, no inhibition of DPP-4 activity by neprilysin was observed (data not shown). Treatment of DPP-4 with diprotin A served as a positive control to demonstrate that DPP-4 activity could be inhibited under the experimental conditions used (Fig. 3c).

\section{Discussion}

We show that in high-fat-fed neprilysin-deficient mice, active GLP-1 levels are elevated compared with in wild-type mice, and are associated with improved beta cell function and glucose tolerance. This is consistent with the loss of neprilysin's ability to cleave and inactivate GLP-1 [10, 11]. Under conditions of prolonged neprilysin deletion, compensatory 


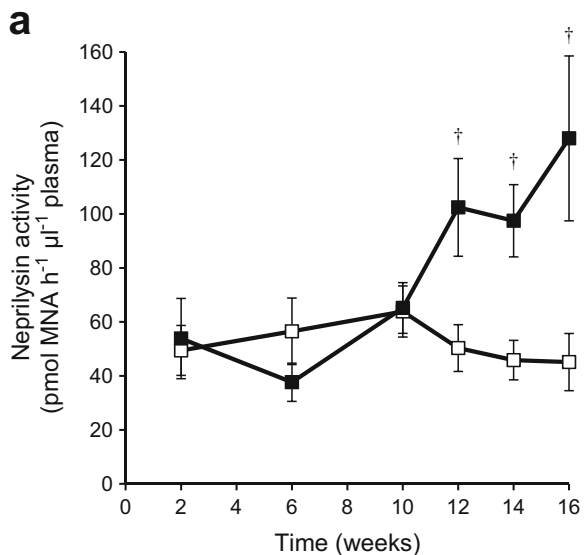

Fig. 2 Neprilysin deficiency reduces DPP-4 activity and enhances active GLP-1 levels in high-fat-fed mice. (a) Plasma neprilysin activity levels in $\mathrm{Nep}^{+/+}$mice over 16 weeks of low- or high-fat feeding. White squares, $\mathrm{Nep}^{+/+} /$low-fat diet; black squares, $\mathrm{Nep}^{+/+} /$high-fat diet. (b, c) Plasma (b)

increases in other proteases that act on the same substrates as neprilysin (e.g. DPP-4) could be expected. However, we observed lower plasma DPP-4 activity in high-fat-fed neprilysin-deficient mice, suggesting that neprilysin may regulate DPP-4 activity and contribute to elevated active GLP-1 levels independent of its direct proteolysis of GLP-1.

Since the possibility cannot be excluded that genetic deletion of neprilysin in mice may have resulted in compensatory changes in the level and/or activity of DPP-4 in vivo, we employed a complementary ex vivo pharmacological

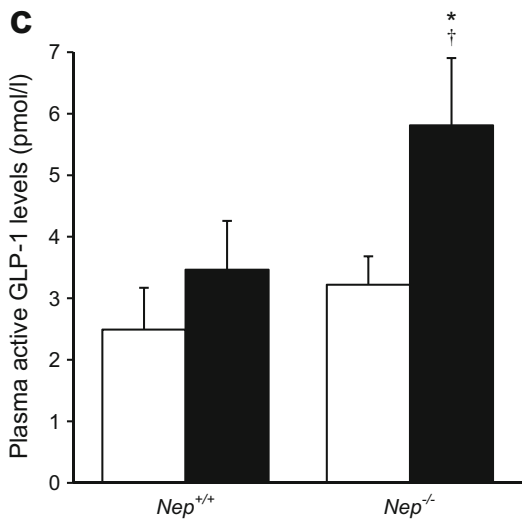

DPP-4 activity and (c) active GLP-1 levels in $\mathrm{Nep}^{+/+}$and $\mathrm{Nep}^{-/-}$mice fed a low- or high-fat diet for 16 weeks. Data are means \pm SEM; $n=9-17$. ${ }^{*} p<0.05 \mathrm{vs} \mathrm{Nep}{ }^{+/+},{ }^{\dagger} p<0.05$ vs the low-fat diet. White bars, low-fat diet; black bars, high-fat diet. MNA, methoxy-2-naphthylamine

approach to determine whether neprilysin can indeed modulate DPP-4 activity. For this, a specific neprilysin inhibitor was added to plasma from wild-type mice and DPP-4 activity measured. Unlike constitutive knockout of neprilysin in mice from birth, pharmacological inhibition has the advantage of eliminating neprilysin activity acutely, thereby decreasing the likelihood of compensatory increases in other proteases and, more specifically, excluding the possibility that changes in DPP-4 expression levels are the mechanism responsible for reduced DPP-4 activity. Strikingly, the effect of acute

Fig. 3 Ex vivo pharmacological inhibition of neprilysin activity reduces DPP-4 activity in plasma from high-fat-fed mice.

(a) Neprilysin and (b) DPP-4 activity levels in plasma treated ex vivo with DL-thiorphan or vehicle from $\mathrm{Nep}^{+/+}$mice fed a low- or high-fat diet for 16 weeks. (c) DPP-4 activity following in vitro incubation of recombinant neprilysin and DPP-4 with DL-thiorphan. Neprilysin:DPP-4 molar ratios of $1: 5$ and 1:10 were tested. Incubation of recombinant neprilysin and DPP-4 with diprotin A served as a positive control. Data are means \pm SEM (a, b) $n=5$ per group. $n=3$ per Neprilysin:DPP-4 molar ratio. White bars, low-fat diet; black bars, high-fat diet. (c) Black bars, 1:5 Neprilysin:DPP-4 molar ratio; White bars, 1:10 Neprilysin:DPP4 molar ratio. $* p<0.05$ vs vehicle, ${ }^{\dagger} p<0.05$ vs low-fat diet. MNA, methoxy-2-naphthylamine
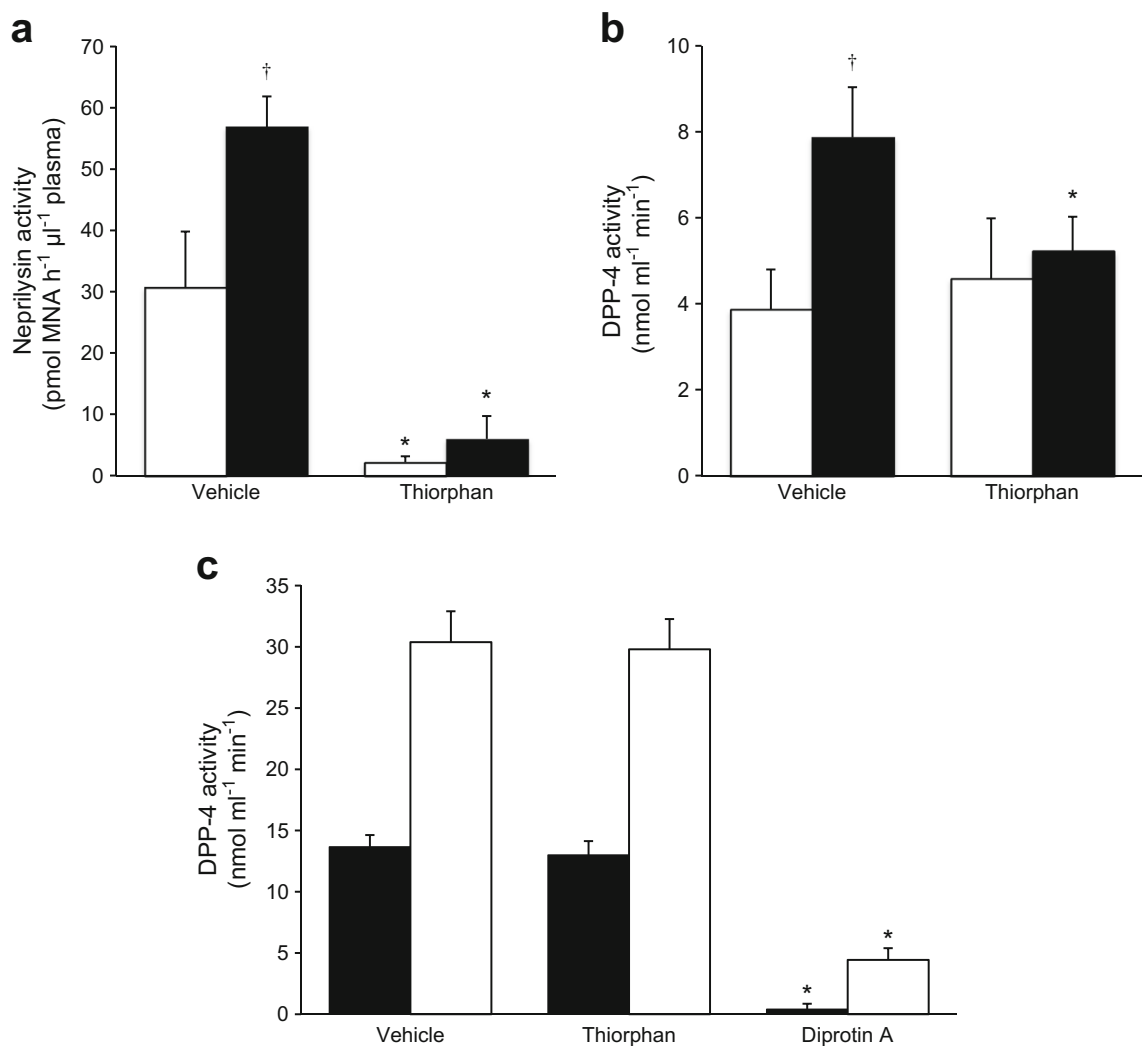
pharmacological neprilysin inhibition on DPP-4 activity was comparable with that seen with genetic ablation of neprilysin, namely, DPP-4 activity in plasma from high-fat-fed but not low-fat-fed mice was reduced when neprilysin activity was abolished. To explore the mechanism responsible for this novel observation, we investigated whether neprilysin directly inhibits DPP-4 by co-incubating recombinant forms of the two proteins in vitro, with or without a neprilysin inhibitor, in a plasma protein free environment that enables retention of enzymatic activity. This paradigm eliminates the possibility of compensation by other proteases present in plasma. Under these in vitro conditions, inhibition of neprilysin failed to affect DPP-4 activity, suggesting that neprilysin does not directly interact with DPP-4. Hence, we postulate that under lowneprilysin/high-fat conditions, there is increased plasma availability of a neprilysin substrate(s) that may act to inhibit DPP-4 activity. While neprilysin substrates are numerous [18], none to our knowledge have been shown to directly modulate DPP-4 activity. Moreover, enzymes such as DPP-4 are typically found to be in excess of their substrates; thus, for a neprilysin substrate to act as a competitive inhibitor, its levels would need to be greater than circulating DPP-4 levels. Another potential mechanism that may explain a reduction in DPP-4 activity with neprilysin inhibition is post-translational modification, specifically hypersialylation of DPP-4. In keeping with this idea, studies have shown that desialylation of DPP-4 increased its activity [19]. Thus, it is possible that a neprilysin substrate promotes sialylation of DPP-4 to downregulate its activity. Further, it has been demonstrated that cytokines are important for regulating DPP-4 activity: IFN $\alpha, \beta$ and $\gamma$ act via STAT $1 \alpha$ signalling to enhance DPP-4 activity [20]. Neprilysin inhibition may interfere with this signalling pathway under high-fat conditions, in which IFN- $\gamma$ production is known to be upregulated [21]. Clearly, future studies are needed to reveal the identity of the neprilysin substrate(s) that may be modulating DPP-4 activity and to assess its suitability as a drug target.

In addition to neprilysin deficiency preserving active GLP-1 levels directly (i.e. lack of neprilysin-mediated proteolysis) or indirectly by lowering DPP-4 activity, it may be possible to further augment active GLP-1 levels by concurrent inhibition of DPP-4. In fact, previous studies showed that when combined with DPP-4 inhibition, short-term pharmacological neprilysin inhibition increased exogenously infused active GLP-1 levels by $40 \%$ and its half-life by sixfold [12]. In terms of whether this translates to a glycaemic benefit in the setting of endogenous GLP-1, a separate study showed that co-administration of a neprilysin inhibitor to diabetic Goto-Kakizaki rats for 12 weeks did not potentiate the antihyperglycaemic effects of DPP-4 inhibition alone [22]. Moreover, the study showed that neprilysin inhibition alone conferred no glycaemic benefit. In contrast, our findings on beta cell function indicate that neprilysin inhibition may improve the glycaemic status of high-fat-fed mice, partly via the incretin effect. Significant differences between the
Goto-Kakizaki rat and neprilysin-deficient mouse models may explain these discrepant findings. Specifically, Goto-Kakizaki rats are lean and develop diabetes when fed a chow/low-fat diet [23], whereas neprilysindeficient mice become obese and insulin resistant when fed a high-fat diet. Indeed, we only saw a beneficial effect of neprilysin ablation on glycaemia under conditions of highfat feeding, including consequent obesity. Of note, body weight, which is typically reduced by GLP-1, was marginally lower in neprilysin-deficient mice despite food intake being comparable with that of wild-type animals; however, high-fatfed neprilysin-deficient mice were still obese relative to lowfat-fed mice. We feel the small difference in body weight between high-fat-fed neprilysin-deficient and wild-type mice is unlikely to have contributed to the marked improvements in glycaemic status.

While our data suggest that increased active GLP-1 levels may contribute to improved glucose metabolism by enhancing beta cell function under conditions of neprilysin deficiency, other mechanisms may also be involved. As neprilysin has broad substrate specificity, cleavage of oligopeptides other than GLP-1 may modulate glycaemic status. For example, increased levels of the neprilysin substrate bradykinin have been shown to contribute to improved insulin sensitivity in neprilysindeficient mice $[5,6]$. Further, blocking bradykinin degradation using a neprilysin inhibitor increased insulin-stimulated glucose uptake at both the whole-body level and in insulin-responsive tissues of Zucker diabetic fatty rats [7]. Thus, neprilysin inhibition is likely to contribute to improved glycaemia via both GLP-1 dependent and independent mechanisms.

In contrast to our findings of improved glycaemic status in high-fat-fed neprilysin-deficient mice, some previous studies have demonstrated that a lack of neprilysin activity confers no glycaemic benefit under conditions of increased dietary fat. For example, in studies of high-fat-fed neprilysin-deficient mice [3, 24], as well as in high-fat-fed wild-type mice or rats treated with a neprilysin inhibitor [25, 26], glucose excursions during an i.p. glucose challenge were no different from those observed in high-fat-fed wild-type control mice or rats. Similarly, as mentioned above, in diabetic Goto-Kakizaki rats, treatment with a neprilysin inhibitor for 12 weeks did not affect oral glucose tolerance [22]. Also, 1-year-old neprilysin-deficient mice with late-onset obesity exhibited impaired oral glucose tolerance [27]. Many of these studies also reported no reduction in body weight with either genetic or pharmacological elimination of neprilysin activity in highfat-fed animals. Data from previous studies may contradict ours because (1) i.p. glucose administration does not stimulate GLP-1 secretion [3, 24-26]; (2) in some cases, C57BL/6J purchased from The Jackson Laboratory were used as controls for neprilysin-deficient mice (rather than being bred from mice heterozygous for the null allele) $[3,24,25]$, or rats were used $[22,26]$; (3) pharmacological inhibition may not 
completely ablate neprilysin activity as is the case with genetic deletion; (4) in 1-year-old neprilysin-deficient mice, glucose tolerance may have been confounded by weight gain due to their increased food intake [27]; and (5) variances in the duration or timing of dietary/inhibitor intervention may result in different outcomes. Reconciling these differences in future work will be critical for developing a better understanding of the role of neprilysin in modulating glucose homeostasis.

In summary, our data raise the interesting possibility that neprilysin inhibition could be beneficial for diabetes patients by increasing active GLP-1 levels via preventing of neprilysin-mediated proteolysis of GLP-1 and/or reducing DPP-4 activity. As neprilysin inhibitors are approved by the US Food and Drug Administration for treating heart failure and their use is well tolerated with no serious adverse events [28], our data argue that further studies are warranted to elucidate the clinical utility of neprilysin inhibitors in type 2 diabetes whether used alone or as an adjunct to other therapies.

Acknowledgements We thank P. Bergquist, C. Braddock, M. Peters and A. Rahman, Seattle Institute for Biomedical and Clinical Research, USA, for their excellent technical support. We also thank D. Drucker and E. Mulvihill, University of Toronto, Canada, for their guidance on measuring plasma DPP-4 activity.

Data availability Authors can confirm that all relevant data are included in the article and/or its supplementary information files.

Funding This work was supported by the National Institutes of Health (grants DK-098506 and DK-080945 to SZ; P30 DK-017047 to the Cell Function Analysis Core, University of Washington Diabetes Research Center) and the United States Department of Veterans Affairs.

Duality of interest statement The authors declare that there is no conflict of interest associated with this manuscript.

Contribution statement SZ conceived the study; JRW and SZ designed the study; BMB and JRW performed experiments; BMB, JRW and SZ analysed the data; SZ wrote the manuscript; and BMB and JRW edited the manuscript. All authors approved the final version to be published. SZ is the guarantor of this work and, as such, had full access to all of the data in the study and takes responsibility for the integrity of the data and the accuracy of the data analysis.

\section{References}

1. Muangman P, Spenny ML, Tamura RN, Gibran NS (2003) Fatty acids and glucose increase neutral endopeptidase activity in human microvascular endothelial cells. Shock 19:508-512

2. Antezana M, Sullivan S, Usui M et al (2002) Neutral endopeptidase activity is increased in the skin of subjects with diabetic ulcers. J Invest Dermatol 119:1400-1404

3. Standeven KF, Hess K, Carter AM et al (2011) Neprilysin, obesity and the metabolic syndrome. Int J Obes (Lond) 35:1031-1040
4. Zraika S, Koh DS, Barrow BM, Lu B, Kahn SE, Andrikopoulos S (2013) Neprilysin deficiency protects against fat-induced insulin secretory dysfunction by maintaining calcium influx. Diabetes 62: 1593-1601

5. Arbin V, Claperon N, Fournie-Zaluski MC, Roques BP, Peyroux $\mathrm{J}$ (2001) Acute effect of the dual angiotensin-converting enzyme and neutral endopeptidase 24-11 inhibitor mixanpril on insulin sensitivity in obese Zucker rat. Br J Pharmacol 133:495-502

6. Arbin V, Claperon N, Fournie-Zaluski MC, Roques BP, Peyroux J (2003) Effects of dual angiotensin-converting enzyme and neutral endopeptidase 24-11 chronic inhibition by mixanpril on insulin sensitivity in lean and obese Zucker rats. J Cardiovasc Pharmacol 41:254-264

7. Wang CH, Leung N, Lapointe N et al (2003) Vasopeptidase inhibitor omapatrilat induces profound insulin sensitization and increases myocardial glucose uptake in Zucker fatty rats: studies comparing a vasopeptidase inhibitor, angiotensin-converting enzyme inhibitor, and angiotensin II type I receptor blocker. Circulation 107:19231929

8. Adelhorst K, Hedegaard BB, Knudsen LB, Kirk O (1994) Structure-activity studies of glucagon-like peptide-1. J Biol Chem 269:6275-6278

9. Mentlein R, Gallwitz B, Schmidt WE (1993) Dipeptidyl-peptidase IV hydrolyses gastric inhibitory polypeptide, glucagon-like peptide-1(7-36)amide, peptide histidine methionine and is responsible for their degradation in human serum. Eur J Biochem 214:829-835

10. Hupe-Sodmann K, Goke R, Goke B et al (1997) Endoproteolysis of glucagon-like peptide (GLP)-1 (7-36) amide by ectopeptidases in RINm5F cells. Peptides 18:625-632

11. Hupe-Sodmann K, McGregor GP, Bridenbaugh R et al (1995) Characterisation of the processing by human neutral endopeptidase 24.11 of GLP-1(7-36) amide and comparison of the substrate specificity of the enzyme for other glucagon-like peptides. Regul Pept 58:149-156

12. Plamboeck A, Holst JJ, Carr RD, Deacon CF (2005) Neutral endopeptidase 24.11 and dipeptidyl peptidase IV are both mediators of the degradation of glucagon-like peptide 1 in the anaesthetised pig. Diabetologia 48:1882-1890

13. Lu B, Gerard NP, Kolakowski LF Jr et al (1995) Neutral endopeptidase modulation of septic shock. J Exp Med 181:2271-2275

14. Terashima H, Okamoto A, Menozzi D, Goetzl EJ, Bunnett NW (1992) Identification of neuropeptide-degrading enzymes in the pancreas. Peptides 13:741-748

15. Matheeussen V, Lambeir AM, Jungraithmayr W et al (2012) Method comparison of dipeptidyl peptidase IV activity assays and their application in biological samples containing reversible inhibitors. Clin Chim Acta 413:456-462

16. Ahmed RH, Huri HZ, Al-Hamodi Z, Salem SD, Muniandy S (2015) Serum levels of soluble CD26/Dipeptidyl Peptidase-IV in type 2 diabetes mellitus and its association with metabolic syndrome and therapy with antidiabetic agents in Malaysian subjects. PLoS One 10, e0140618

17. Lamers D, Famulla S, Wronkowitz N et al (2011) Dipeptidyl peptidase 4 is a novel adipokine potentially linking obesity to the metabolic syndrome. Diabetes 60:1917-1925

18. Bayes-Genis A, Barallat J, Richards AM (2016) A test in context: neprilysin: function, inhibition, and biomarker. J Am Coll Cardiol 68:639-653

19. Cuchacovich M, Gatica H, Pizzo SV, Gonzalez-Gronow M (2001) Characterization of human serum dipeptidyl peptidase IV (CD26) and analysis of its autoantibodies in patients with rheumatoid arthritis and other autoimmune diseases. Clin Exp Rheumatol 19:673-680

20. Bauvois B, Djavaheri-Mergny M, Rouillard D, Dumont J, Wietzerbin J (2000) Regulation of CD26/DPPIV gene expression by interferons and retinoic acid in tumor B cells. Oncogene 19:265-272 
21. Wensveen FM, Jelencic V, Valentic S et al (2015) NK cells link obesity-induced adipose stress to inflammation and insulin resistance. Nat Immunol 16:376-385

22. Simonsen L, Pilgaard S, Carr RD, Kanstrup AB, Holst JJ, Deacon CF (2009) Inhibition of neutral endopeptidase 24.11 does not potentiate the improvement in glycemic control obtained with dipeptidyl peptidase-4 inhibition in diabetic Goto-Kakizaki rats. Horm Metab Res 41:851-853

23. Akash MS, Rehman K, Chen S (2013) Goto-Kakizaki rats: its suitability as non-obese diabetic animal model for spontaneous type 2 diabetes mellitus. Curr Diabetes Rev 9:387-396

24. Davidson E, Coppey L, Lu B et al (2009) The roles of streptozotocin neurotoxicity and neutral endopeptidase in murine experimental diabetic neuropathy. Exp Diabetes Res 2009:431980
25. Coppey L, Lu B, Gerard C, Yorek MA (2012) Effect of inhibition of angiotensin-converting enzyme and/or neutral endopeptidase on neuropathy in high-fat-fed C57B1/6J mice. J Obes 2012:326806

26. Davidson EP, Coppey LJ, Dake B, Yorek MA (2011) Effect of treatment of Sprague Dawley rats with AVE7688, enalapril, or candoxatril on diet-induced obesity. J Obes 2011:686952

27. Becker M, Siems WE, Kluge R et al (2010) New function for an old enzyme: NEP deficient mice develop late-onset obesity. PLoS One $5, \mathrm{e} 12793$

28. McMurray JJ, Packer M, Desai AS et al (2014) Angiotensinneprilysin inhibition versus enalapril in heart failure. $\mathrm{N}$ Engl J Med 371:993-1004 